\title{
Pediatric meningococcocal meningitis in the acute phase: how much does it cost?
}

\author{
Elena Bozzola ${ }^{{ }^{*}} \mathbb{D}$, Stefano Guolo ${ }^{2}$, Enea Bonci ${ }^{3}$, Chiara Rossetti ${ }^{1}$, Mauro Bozzola ${ }^{4}$, Massimiliano Raponi ${ }^{2}$ and \\ Alberto Villani ${ }^{1}$
}

\begin{abstract}
Background: Meningococcal meningitis (MM) is known to be responsible of high cost for the Public Health Administration. Aim of the work is to calculate the costs for the hospitalization of pediatric patients affected by MM.

Methods: We calculate the costs for the hospitalization of pediatric patients affected by MM in the acute phase (HAP) over a nine year period. We performed a MEDLINE search to verify the cost of MM HAP reported in other studies.

Results: At Bambino Gesù Children Hospital, the median cost of HAP was of 12,604 euro (range from 9203 to 35,050 euro). Comparing our data with the previous studies, we find out similar results of approximately 16,750 euro (range 12,000-20,000 euro).

Discussion: Despite the relative rarety of the disease, MM is associated to direct high cost of HAP.

Conclusions: Hospital costs are an important end-point in health economic evaluation of the disease and may be useful to policy makers and health economists to understand the potential benefit of improving meningococcal vaccination programmes.
\end{abstract}

Keywords: Meningitis, Hospital, Cost, Children

\section{Background}

Neisseria meningitidis $(\mathrm{Nm})$ is the causative agent of meningococcal meningitis (MM).

Among the 13 subgroups of $\mathrm{Nm}$, six of them $(\mathrm{A}, \mathrm{B}, \mathrm{C}, \mathrm{W} 135, \mathrm{X}, \mathrm{Y})$ may cause clinical disease in humans and may be responsible of mortality and morbidity among survivors. [1-3]

In Italy, the incidence of MM is of 0.3 cases per year per 100,000 habitants, according to the Italian National Institute of Public Health. In the pediatric age, the highest incidence is among children aged less than 4 years. In particular, in infants younger than 1 year, 4 cases per year per 100,000 had been notified. [4]

$\mathrm{MM}$ is also responsible of high cost for the acute care and for the follow-up of affected patients. [5-7] To date, vaccination constitutes the best preventive strategies

\footnotetext{
*Correspondence: elena.bozzola@opbg.net

${ }^{1}$ Pediatric and Infectious Disease Unit, Bambino Gesù Children Hospital,

Rome, Italy

Full list of author information is available at the end of the article
}

against $\mathrm{MM}$ and it can protect against subgroups A, B, C, $\mathrm{Y}$ and W135. In Italy, antimeningoccocal vaccines are currently included in the routine childhood vaccination strategies, but they are not compulsory. [8] The first meningococcal conjugate vaccine was against serogroup $\mathrm{C}$ and was introduced in the Italian national immunization plan in 2005. A significant reduction in the incidence of invasive meningococcal disease in the post vaccination period compared to the pre-vaccination one has been observed in Italy. $[9,10]$ The actual vaccination coverage against $\mathrm{Nm} \mathrm{C}$ is of $80,67 \%$ at 24 months of life. The coverage against $\mathrm{Nm} \mathrm{B}$ is very low as just $14,72 \%$ of 2 years old children received the immunization. Finally, 18,28\% of 2 years old children received the vaccine against $\mathrm{Nm}$ ACW135Y.

Aim of the study is to estimate the costs of MM hospitalization in the acute phase (HAP) in an Italian pediatric population.

(C) The Author(s). 2019 Open Access This article is distributed under the terms of the Creative Commons Attribution 4.0 International License (http://creativecommons.org/licenses/by/4.0/), which permits unrestricted use, distribution, and reproduction in any medium, provided you give appropriate credit to the original author(s) and the source, provide a link to the Creative Commons license, and indicate if changes were made. The Creative Commons Public Domain Dedication waiver (http://creativecommons.org/publicdomain/zero/1.0/) applies to the data made available in this article, unless otherwise stated. 


\section{Material and methods}

The study was performed at Bambino Gesù Children Hospital, which is a tertiary pediatric hospital in Rome, Lazio, Italy. The records of patients aged $<18$ years admitted at hospital for MM were analyzed from January 2006 till January 2015. The MM cases were identified using the international classification of diseases, ninth revision, clinical modification (ICD-9-CM) which assigns numeric codes to diagnosis. ICD-9 code which identifies MM is 036.0.

The medical records of patients with principal discharge ICD-9-CM code 036.0 were analyzed in order to verify the laboratory-confirmed diagnosis. If the cerebrospinal fluid antigen and/or the culture was positive for $\mathrm{Nm}$, the patient entered the study.

Patients who did not fulfill the inclusion criteria were excluded from the study. As for the others, direct medical costs were extracted from the Lazio Regional Health Service Tariffs and included: cost of hospital accommodation and management at the Infectious Diseases Unit and/or at the Intensive care Unit. An important part of hospitalization is caused by the cost of each single day of hospitalization. The price per day of hospital stay is different between the Departments of Infectious Diseases and of Intensive Care. This is due to the strong difference in care complexity of the two departments. We considered a daily cost of $€ 626$ for the intensive care unit, defined as a very high complexity unit and $€ 476$ for the infectious diseases, defined as a low complexity unit.

To this cost, the price of procedures (imaging, laboratory exams, medical and paramedical evaluations), medical and surgical treatment were added. The appropriate procedure codes were applied, in order to evaluate the single cost of any exam and therapy.

We performed a MEDLINE search to calculate the cost of MM HAP. For the purpose of this study, we used the keywords "Meningitis, Meningococcal"[Mesh] AND "Costs and Cost Analysis"[Mesh] OR "Hospital Costs"[Mesh] OR "Employer Health Costs"[Mesh] OR "Health Care Costs"[Mesh] OR "Drug Costs"[Mesh] OR "Cost of Illness"[Mesh] OR "Direct Service Costs"[Mesh] OR "Health Expenditures"[Mesh] OR “Cost-Benefit Analysis"[Mesh] OR "Economics"[Mesh]. The search concerned English publications referring to patients younger than 18 years of age, from January 2006 to December 2016.

We included in the search cases of pediatric laboratory-confirmed MM with precise cost of HAP. We excluded reports: [1] if they concerned meningitis with undistinguished or unconfirmed etiology; [2] if they concerned adults; [3] if they presented undistinguished data on both adults and children; [4] if they report the undistinguished cost of HAP and of follow-up.

From all reports, we extracted both the number of $\mathrm{MM}$ and the mean cost of HAP.

\section{Results}

Among all the children hospitalized in the study period, 32 fulfilled the inclusion criteria. The mean age of patients was of 5.3 years (range from 40 days to 16.5 years, median 4 years). The proportion of male and female was similar (53\% males and 47\% female). All the patients had neither an underlying medical condition nor received previous anti-meningococcal vaccination.

Among enrolled patients, subgroup $\mathrm{C}$ has been isolated in 7 cases, subgroup B in 9, subgroup $Y$ in 3 and subgroup W135 in 1 patient. In 12 cases no subgroup has been detached.

The median cost of HAP was of 12,604 euro (range from 9203 to 35,050 euro; mean cost of 14,874 euro).

Medical condition both at MM onset and during hospitalization may have complicated the disease, requiring a prolonged length stay, further exams and therapies.

Table 1 summarizes the costs for each patients.

The mean HAP of the six patients aged $<1$ year was of 17,306 euro, higher than those of the 12 children aged 1 to 5 years (13,313 euro), as well as of the eight cases aged 5 to 10 years $(15,025$ euro), and of the six patients older than 10 years (14,059 euro).

Patients had been hospitalized for a mean time of 17 days (range from 11 to 25 days). Fourteen children required Intensive care Unit assistance for a mean period of 3 days (range from 24 to $189 \mathrm{~h}$ ), which contributes to the increment of the hospitalization costs.

Patients requiring Intensive Unit Care had higher HAP (17,931 euro) than those hospitalized at Infectious Diseases Unit (12,496 euro).

The HAP during the period study did not change.

Out of 16 reports resulting from our MEDLINE analysis, just 2 manuscripts matched the criteria for inclusion in the study. No Italian manuscript matched the inclusion criteria. The manuscript were carefully examined to estimate HAP. $[5,6]$

The other 14 manuscripts were excluded because they: [1] concern costs on vaccine and vaccination programmes [7]; [2] report costs only on diagnostic tools [1]; [3] reported indistinguishable data on the adult and pediatric populations [2]; [6] reported only clinical data [1]; [7] reported not real cost of HAP but scenario [2, 8] reported data from other sources [1].

Comparing our data with the selected studies, we find out similar results of approximately 16,750 euro (range 12,000-20,000 euro). [6, 7]

\section{Discussion}

In our study, we reported the cost of MM HAP. Hospital costs are an important end-point in health economic evaluation of the disease.

We analyzed cost associated with inpatient servicies in the acute phase which are one of the major component 
Table 1 HAP cost for each patients (in euro)

\begin{tabular}{|c|c|c|c|c|c|c|c|c|}
\hline Patient & SEX & AGE & HAP costs & Intensive care costs & Laboratory costs & Instrumental costs & Specialist visits costs & Therapy costs \\
\hline 1 & $F$ & 0,09 & 9203 & 0 & 843 & 223 & 21 & 26 \\
\hline 2 & $\mathrm{~F}$ & 9,9 & 9475 & 0 & 671 & 167 & 41 & 122 \\
\hline 3 & M & 3,98 & 9533 & 1236 & 865 & 207 & 62 & 69 \\
\hline 4 & M & 3,39 & 9545 & 0 & 683 & 243 & 41 & 42 \\
\hline 5 & M & 9,82 & 9638 & 1050 & 761 & 144 & 41 & 136 \\
\hline 6 & $\mathrm{~F}$ & 1,92 & 9740 & 0 & 874 & 342 & 41 & 196 \\
\hline 7 & $\mathrm{~F}$ & 0,56 & 10,094 & 0 & 871 & 309 & 62 & 53 \\
\hline 8 & $\mathrm{~F}$ & 8,25 & 10,224 & 0 & 689 & 266 & 41 & 84 \\
\hline 9 & $\mathrm{~F}$ & 7,28 & 10,320 & 0 & 689 & 278 & 83 & 101 \\
\hline 10 & M & 1,27 & 10,372 & 0 & 817 & 509 & 41 & 66 \\
\hline 11 & $M$ & 3,99 & 10,930 & 741 & 947 & 179 & 124 & 104 \\
\hline 12 & $M$ & 6,6 & 11,526 & 0 & 816 & 412 & 165 & 83 \\
\hline 13 & F & 0,53 & 11,565 & 0 & 944 & 200 & 41 & 35 \\
\hline 14 & $F$ & 1,09 & 11,696 & 2317 & 979 & 98 & 83 & 43 \\
\hline 15 & $M$ & 15,46 & 11,708 & 0 & 748 & 98 & 41 & 148 \\
\hline 16 & $F$ & 0,8 & 11,958 & 0 & 832 & 243 & 41 & 32 \\
\hline 17 & $\mathrm{~F}$ & 16,51 & 13,250 & 3274 & 816 & 278 & 83 & 319 \\
\hline 18 & $M$ & 15,3 & 13,783 & 2224 & 962 & 290 & 83 & 181 \\
\hline 19 & F & 1,26 & 14,528 & 2749 & 1016 & 332 & 83 & 433 \\
\hline 20 & F & 1,99 & 14,809 & 0 & 835 & 230 & 83 & 86 \\
\hline 21 & M & 11,22 & 15,069 & 3700 & 936 & 274 & 62 & 141 \\
\hline 22 & F & 4,01 & 15,267 & 0 & 748 & 266 & 83 & 108 \\
\hline 23 & $M$ & 1,23 & 15,595 & 0 & 689 & 573 & 41 & 41 \\
\hline 24 & $M$ & 2,09 & 15,757 & 0 & 959 & 278 & 83 & 132 \\
\hline 25 & $M$ & 11,67 & 16,486 & 2440 & 827 & 1130 & 248 & 259 \\
\hline 26 & $M$ & 5,23 & 16,754 & 0 & 746 & 972 & 145 & 83 \\
\hline 27 & M & 0,6 & 21,022 & 0 & 869 & 713 & 269 & 76 \\
\hline 28 & M & 2,36 & 21,993 & 2224 & 634 & 376 & 62 & 7659 \\
\hline 29 & $F$ & 0,5 & 26,309 & 5838 & 1212 & 407 & 186 & 3260 \\
\hline 30 & F & 9,86 & 26,352 & 2687 & 1085 & 572 & 207 & 9034 \\
\hline 31 & $M$ & 6,06 & 26,423 & 5190 & 1014 & 278 & 124 & 9329 \\
\hline 32 & M & 7,19 & 35,050 & 4448 & 1050 & 584 & 124 & 13,542 \\
\hline
\end{tabular}

of health care utilization in the management of MM. $[11,12]$

The cost of the acute phase of the disease with a high morbidity among survivors may constitute the tip of the iceberg of the disease. [5]

We demonstrated that $\mathrm{MM}$ is associated with direct health cost as for a median of 12,604 euro each patient. Despite the relative rarety of MM, the high cost of HAP translate to a consistent economic burden. In fact, we have also to consider indirect costs, such as missing working days for parents who assist children (productivity loss) and of prophylaxis to close contacts of the index case, to prevent outbreaks. Moreover, also medical and paramedical equipment who should be instructed to manage MM represent an indirect cost.

Numerous studies estimating the costs of meningococcal disease have previously been performed. Nevertheless most studies used ICD diagnosis codes not verified against laboratory results or hospital records. [11, 12] A possible consequence is an under or over estimation of hospital costs. Moreover, other studies did not differentiate costs among the acute phase and lifelong costs of treatment and management of invalidating sequelae. [5] Few studies are available only on MM HAP. [6, 7]

Potential limits of our study is the small sample size and that all data were collected from a single pediatric 
institution. Finally, a limitation of the comparing analysis is that the previous works did not report the single cost items, so that the result is approximate and may be influenced by a different approach to acute patient.

\section{Conclusions}

In conclusion, we conducted this analysis as we think that financial studies are important to evaluate the cost saving in order to public funding decisions like immunization strategies and vaccination programmes. Economic evaluation, including length of hospitalization, exams and treatments, may be useful to policy makers and health economists to understand the potential benefit of improving meningococcal vaccination programmes.

\section{Abbreviations \\ HAP: hospitalization in the acute phase; MM: meningococcal meningitis; Nm: Neisseria meningitidis}

\section{Acknowledgments}

Not applicable.

\section{Funding}

Nothing to declare.

\section{Availability of data and materials}

Bambino Gesù Children Hospital, study-office of Dr. Bozzola.

\section{Authors' contributions}

EB coordinated the study; SG and AV designed the study; CR collected the data, MR analyzed the results, EB and MB performed the Medline search. All authors read and approved the final version.

\section{Ethics approval and consent to participate}

Not applicable.

\section{Consent for publication}

Not applicable.

\section{Competing interests}

The authors declare that they have no competing interests.

\section{Publisher's Note}

Springer Nature remains neutral with regard to jurisdictional claims in published maps and institutional affiliations.

\section{Author details}

${ }^{1}$ Pediatric and Infectious Disease Unit, Bambino Gesù Children Hospital, Rome, Italy. 'Sanitary Direction, Bambino Gesù Children Hospital, Rome, Italy. ${ }^{3}$ Sperimental Medicine Department, La Sapienza University, Rome, Italy. ${ }^{4}$ Internal Medicine and Therapeutics Department, Pediatrics and Adolescentology Unit, University of Pavia, Pavia, Italy.

Received: 27 July 2018 Accepted: 3 February 2019

Published online: 13 February 2019

\section{References}

1. Harrison LH, Mohan N, Kirkpatrick P. Meningococcal group A, C, Y and W135 conjugate vaccine. Nat Rev Drug Discov. 2010;9(6):429-30.

2. Viner RM, Booy R, Johnson H, Edmunds WJ, Hudson L, Bedford H, Kaczmarski E, Rajput K, Ramsay M, Christie D. Outcomes of invasive meningococcal serogroup B disease in children and adolescents (MOSAIC): a case-control study. Lancet Neurol. 2012:11(9):774-83.

3. Gianchecchi E, Torelli A, Piccini G, Piccirella S, Montomoli E. Neisseria meningitidis infection: who, when and where? Expert Rev Anti-Infect Ther 2015;13(10):1249-63.
4. Epicentro. www.epicentro.iss.it

5. Darbà J, Kaskens L, Hark M, Wright C. Costs of surviving meningococcal disease in Spain: evaluation for two cases of severe meningitis and septicaemia. Vaccine. 2014 Sep 3:32(39):5006-12.

6. O'Brien JA, Caro JJ, Getsios D. Managing meningococcal disease in the United States: Hospital case characteristics and costs by age. Value Health. 2006 Jul-Aug;9(4):236-43.

7. Wang B, Haji Ali Afzali H, Marshall H. The inpatient costs and hospital service use associated with invasive meningococcal disease in South Australian children. Vaccine. 2014;32(37):4791-8.

8. Sadarangani M, Pollard AJ. Can we control all-cause meningococcal disease in Europe? Clin Microbiol Infect. 2016 pii: S1198-743X(16)30021-0.

9. de Waure C, Miglietta A, Nedovic D, Mereu G, Ricciardi W. Reduction in Neisseria meningitidis infection in Italy after Meningococcal C conjugate vaccine introduction: A time trend analysis of 1994-2012 series. Hum Vaccin Immunother 2016;12(2):467-473.

10. Stefanelli P, Fazio C, Neri A, Boros S, Renna G, Pompa MG. National Surveillance System Collaborative Centers. Changing epidemiology of Infant Meningococcal Disease after the introduction of meningococcal serogroup C vaccine in Italy, 2006-2014. Vaccine. 2015;33(31):3678-81.

11. Davis KL, Misurski D, Miller J, Karve S. Cost impact of complications in meningococcal disease: evidence from a United States managed care population. Hum Vaccin. 2011 Apr;7(4):458-65.

12. Davis KL, Bell TJ, Miller JM, Misurski DA, Bapat B. Hospital costs, length of stay and mortality associated with childhood, adolescent and young adult meningococcal disease in the US. Appl Health Econ Health Policy. 2011;9(3): 197-207.

\section{Ready to submit your research? Choose BMC and benefit from:}

- fast, convenient online submission

- thorough peer review by experienced researchers in your field

- rapid publication on acceptance

- support for research data, including large and complex data types

- gold Open Access which fosters wider collaboration and increased citations

- maximum visibility for your research: over $100 \mathrm{M}$ website views per year

At BMC, research is always in progress.

Learn more biomedcentral.com/submissions 